\title{
Visual Attention Modulates Insight Versus Analytic Solving of Verbal Problems
}

\author{
Ezra Wegbreit', Satoru Suzuki², Marcia Grabowecky'2, John Kounios ${ }^{3}$, and \\ Mark Beeman ${ }^{2}$
}

\begin{abstract}
:
Behavioral and neuroimaging findings indicate that distinct cognitive and neural processes underlie solving problems with sudden insight. Moreover, people with less focused attention sometimes perform better on tests of insight and creative problem solving. However, it remains unclear whether different states of attention, within individuals, influence the likelihood of solving problems with insight or with analysis. In this experiment, participants $(\mathrm{N}=40)$ performed a baseline block of verbal problems, then performed one of two visual tasks, each emphasizing a distinct aspect of visual attention, followed by a second block of verbal problems to assess change in performance. After participants engaged in a center-focused flanker task requiring relatively focused visual attention, they reported solving more verbal problems with analytic processing. In contrast, after participants engaged in a rapid object identification task requiring attention to broad space and weak associations, they reported solving more verbal problems with insight. These results suggest that general attention mechanisms influence both visual attention task performance and verbal problem solving.
\end{abstract}

\section{Keywords:}

verbal problem solving, visual attention, insight, creativity, focused attention, broadened attention

\footnotetext{
${ }^{1}$ University of Illinois at Chicago; ${ }^{2}$ Northwestern University; ${ }^{3}$ Drexel University
} 


\section{Introduction}

From mastering fire and developing agriculture to producing advanced microchips and landing on the moon, human beings are the world's foremost problem solvers. Yet sometimes, people become stuck on a problem-whether it is seemingly easy, or difficult but solvable. Then, after the initial impasse, the solution comes to them with a sudden insight, often accompanied by an "Aha!" experience. Insightful solutions to problems have inspired a large body of anecdotes, and perhaps some mythology (Weisberg, 1986). Nearly a century of scientific research has investigated the cognitive processes that support solving problems with insight (Duncker, 1945; Maier, 1931; for review, see Bowden, Jung-Beeman, Fleck, \& Kounios, 2005; Sternberg \& Davidson, 1995). Recent advances have allowed researchers to begin investigating the neural processing involved in insight solutions (e.g. Jung-Beeman et al., 2004; Kounios et al., 2006), as well as examining the contribution of other general processes, such as mood and attention, to insight solutions (Rowe, Hirsh, \& Anderson, 2007; Subramaniam, Kounios, Parrish, \& Jung-Beeman, 2009).

Several characteristics differentiate insight solutions from analytic ones. Most researchers agree that analytic solving involves methodical, strategic, step-by-step processing, where each (successful) step reduces the distance to the goal. Although solving may take time or be difficult, the steps involved are generally known or deducible, and people gradually approach solution (Metcalfe \& Wiebe, 1987). In contrast, insight solutions are sudden and obvious (Bowden et al., 2005). Not only are the steps necessary to solve unknown at the beginning of the problem, but even after solution, solvers may be unable to report the processes that led to it (Schooler, Ohlsson, \& Brooks, 1993). Insights often involve restructuring or reframing a person's representation of the problem, and they can occur after a person has experienced a period of impasse wherein no progress is being made toward solving the problem (Schooler \& Melcher, 1995).

Although psychologists often contrast specific "insight problems" with "analytic problems," many of these classified problems can be solved either with insight, with analytic processing, or with a little of both (Bowden et al., 2005). Thus, insight is not a type of problem in itself, but is a method by which people can solve a problem. A number of experiments have demonstrated that participants' subjective judgments of solution type have been correlated with objective measures. For instance, participants show different patterns of semantic priming when recognizing solutions that evoke a subjective insight experience and those that do not evoke an insight experience (Bowden \& Jung-Beeman, 2003b). Furthermore, participants show distinct neural correlates when they report solving a problem with insight versus when they report solving a problem with analysis (Jung-Beeman et al., 2004; Kounios et al., 2006; Subramaniam et al., 2009). In addition, before participants even see a problem, they show different neural patterns for a problem eventually solved with insight versus one that they will eventually solve by analysis (Kounios et al., 2006). Finally, resting state brain activity measured by EEG differs 
between participants who later report solving most anagrams with insight versus those who report solving most anagrams analytically (Kounios et al., 2008). Similarly, distinct patterns are observed if objective criterion, such as time to solution, is used in conjunction with reported solving (Aziz-Zadeh et al., 2009).

The current experiment examines whether visual attention tasks alter participants' tendency to solve problems with self-reported insight or analysis. Attention is one of several factors thought to affect the ability to solve problems with insight, creativity, or cognitive flexibility, within and across individuals (Ansburg, 2000; Ansburg \& Hill, 2003). Some states of attention appear particularly conducive to insight solving, and other states of attention appear conducive to analytic solving. Attention can vary across individuals (i.e. attentional traits) and within individuals (i.e. attentional states) and both can affect the manner in which people approach and solve problems (Ansburg \& Hill, 2003; Friedman, Fishbach, Förster, \& Werth et al., 2003).

People who are more distractible often have an advantage for problems thought to require insight or cognitive flexibility, such as anagrams and word problems like the Remote Associates Test (RAT; Mednick \& Mednick, 1967). For example, people who have more difficulty ignoring distraction on selective attention tasks tend to solve problems amenable to insight better than problems amenable to incremental, step-by-step analysis (Ansburg \& Hill, 2003; Mendelsohn \& Griswold, 1966; Mendelsohn \& Lindholm, 1972). Participants who exhibit more trouble filtering out distracting stimuli while completing selective attention tasks also perform better on open-ended tests of creativity such as sorting objects and decoding pattern meanings (Dykes \& McGhie, 1976; Rawlings, 1985), completing unfinished drawings (Necka, 1999), and writing creative poems (Kasof, 1997). Finally, people who show reduced ability to screen out irrelevant stimuli from current attentional focus also report more real-world creative achievements than people who show greater ability to screen out irrelevant stimuli (Carson, Peterson, \& Higgins, 2003). Thus, people who have more "leaky" attentional filters show better performance on tasks designed to measure creativity and show more creativity in the real world, yet this attentional broadening comes with a cost when perceptual focus is required.

A recent study provides some neural evidence for the possibility that individual differences in attentional traits are associated with the tendency to solve either with insight or with analysis (Kounios, et al., 2008). In this study, resting-state EEG was acquired before the participants even knew the task that they were about to perform. Participants who would later solve more anagrams via (self-reported) insight showed less occipital alphaband activity, reflecting less inhibition of the visual attention system and a trait toward employing more diffuse attentional states. In contrast, participants who later solved more anagrams via analysis showed higher beta activity in their occipital lobes, suggesting they tend to employ more focused attention states (Kounios, et al., 2008). These results also 
suggest that individual differences in neural activation reflect default attentional styles that influence the methods which participants later employ to solve problems.

One explanation for these phenomena is that people with trouble filtering out distracting stimuli cast a wider attentional net (Dykes \& McGhie, 1976) and they are more likely to notice external stimuli "opportunistically" (Seifert, Meyer, Davidson, Patalano, \& Yaniv, 1995), or to notice internal weakly-activated associations that may later become relevant to solving an open-ended problem with creative or insightful processing. As evidence for this hypothesis, participants who have trouble filtering out irrelevant words on a focused attention task are better able to utilize the irrelevant words as solution cues on a later anagram task, even when verbal intelligence (Mendelsohn \& Griswold, 1966; Mendelsohn \& Lindholm, 1972) and overall anagram performance (Ansburg \& Hill, 2003) are controlled for statistically. These participants are not just distracted by the irrelevant stimuli; they are also better able to use the stimuli once they became relevant to the anagram task. Thus, individual differences between participants' preferred attentional states affect the likelihood and method by which they solve problems using insight processing.

Differences in mood or affect are also related to solving problems with creative insights, and this may provide indirect evidence for the role of attention. Although the link between positive affect and creativity is not always straightforward (Kaufmann, 2003; Kaufmann \& Vosburg, 1997), generally people who are in a positive mood produce more novel responses on divergent thinking tasks (Isen, 1999; Gasper, 2003, 2004b) and solve more remote-associates type problems (Isen, Daubman, \& Nowicki, 1987; Subramaniam et al., 2009) than people who are in a negative or neutral mood.

We believe that affect modulates problem solving through attention, in part because people who are in a positive mood tend to employ a broader focus of attention than people in negative and neutral moods (Gasper \& Clore, 2002; Gasper, 2004a). In one recent study, when participants were induced into a positive mood they showed both spatially broadened visual attention and enhanced problem solving performance on the RAT, and when they were induced into a sad or a neutral mood they showed more focal visual attention and relatively diminished problem solving performance on the RAT (Rowe et al., 2007).

Further evidence that attention (or cognitive control) mediates the links between mood and problem solving comes from a recent $\mathrm{FMRI}$ study of the anatomical substrate of mood and peoples' use of insight processing in problem solving. Participants high in positive mood prior to the experiment solved more remote-associate word problems overall, and more with insight, than participants in a less positive mood. A conjunction analysis of brain areas involved in both mood and insightful problem solving revealed that the anterior cingulate cortex (ACC) was sensitive both to the participants' mood state and to their subsequent solution style. The study suggests that positive mood enhances insight problem solving by modulating ACC activity, affecting attentional and cognitive

- volume 4, no. 2 (Spring 2012) 
control mechanisms putatively involved in detecting nondominant solution candidates (Subramaniam et al., 2009).

Furthermore, activity in this ACC area modulated by mood also seems to indicate whether people are in a mental state conducive to solving with insight. Prior to seeing each problem, participants show increased activity in the ACC before trials that they will eventually solve with insight (Kounios et al., 2006). The ACC is known to be involved in increasing top-down control of attention (Kerns et al., 2004) and in tuning one's attentional focus to attend to broader or narrower spatial extents (Weissman, Gopalakrishnan, Hazlett, \&Woldorff, 2005). In addition, activity levels in the ACC may index how ready participants are to detect and to switch attention to less common conceptual associates, if the most prominent associate is not the solution (Subramaniam et al., 2009). Thus, participants' attentional state just prior to working on a verbal problem can affect the method by which they solve the problem.

Beyond correlating individual differences in attentional style with participants' performance on creativity tasks, and showing that changes in mood affect both attention and insight (Rowe et al., 2007), a few researchers have also attempted to manipulate participants' attentional states in order to affect their performance on subsequent creativity tasks (Friedman et al, 2003). In theory, participants induced to have a broad or narrow scope of perceptual attention could subsequently have a similarly broad or narrow scope of conceptual attention, and having a broader or narrower scope of conceptual attention would lead to enhanced or diminished creativity, respectively. As evidence for this proposition, people who were asked to search within or focus upon a relatively broad area produced more unusual uses for an object and more creative category exemplars than participants asked to attend to a narrower spatial extent (Friedman et al., 2003).

Previous work has thus manipulated attention indirectly or contrasted open-ended problems that are most likely to be solved creatively with closed-ended problems that are most likely to be solved analytically (e.g. algorithmically). In the present study, participants completed Compound Remote Associate (CRA) problems (Bowden \& Jung-Beeman, 2003b), closed-ended word problems that can be solved either analytically or with insight. In each CRA problem, participants saw three problem words (e.g. carbon, cat, right) with the goal of finding one word (e.g. "copy") that forms a compound word or a common two-word phrase with all of the problem words (carbon copy, copycat, copyright). Thus, the type of problem and general processing demands were equated across insight and analytic solutions.

Although solutions by analysis and insight involve overlapping processes (Ansburg, 2000), several processes are unique to each type of solving. Participants often report that they begin each problem by thinking about possible compound words that will fit one or more of the problem words (e.g. "carbon paper", "catcall", or "right angle" for the example 
problem shown above). Some participants may strategically choose to focus on the word that they feel is likely to have the most compound words, in order to have a large problem space in which to search. Alternatively, participants may choose to focus on the word that they feel is likely to have the fewest compound words in order to restrict their search deliberately to narrow down the possibilities. If participants solve a problem using one of these search algorithms they are likely to report that they solved the problem via analysis because they were able to report that they were getting closer and closer to the solution by eliminating alternatives, a hallmark feature of solving problems by analysis (Metcalfe \& Wiebe, 1987).

However, problems such as the CRA problems that entail a large problem space and potentially a "narrow canyon of exploration" are also likely candidates to be solved via insight (Perkins, 2001). If participants become lost in the associations of a huge problem space then they are likely to become stuck and then suddenly "stumble upon" the correct solution such that it comes to them in a flash. Similarly, if participants become mired in a "canyon" then they might suddenly break free by activating a more remote associate of a word (e.g. realizing that the word "bank" could also be paired with "river", rather than just "money"). As evidence for this supposition, participants who report solving a problem with insight show greater priming in the right hemisphere, known to be more sensitive to remote associates, than the left hemisphere (Beeman \& Bowden, 2000; Bowden \& JungBeeman, 2003a). Thus, insight solutions to CRA problems involve more sudden activations of associations that are remote to those currently in the focus of attention.

While conscious search activity is taking place, activation related to each of the three words spreads throughout the semantic association network (Beeman \& Bowden, 2000; Bowden \& Jung-Beeman, 2003a). These CRA problems are designed such that this network of associations has one and only one semantic convergence that satisfies the criteria of the problem (Bowden \& Jung-Beeman, 2003b). However, participants may activate this node without realizing it, particularly if their covert "inner voice" is involved in consciously searching through the realm of possibilities, similar to the way that overt verbalization impairs solving problems via insight (Schooler, Ohlsson, \& Brooks, 1993). Thus, in order to solve a CRA problem via insight, participants presumably need to detect the weak solution-related subthreshold activation and switch their attention to this weak signal amidst the noise of competing semantic activation. If participants suddenly detect activation at this "convergence node" in their semantic network (Jung-Beeman, 2005), then they are likely to report having solved the problem via insight (Jung-Beeman et al., 2004). Given the earlier neural findings that participants activate different areas of the attention system (i.e. the $\mathrm{ACC}$ ) in different ways depending on the type of processing that they use (Kounios et al., 2006; Subramaniam et al., 2009), manipulating participants' attentional focus could subsequently alter the processing by which participants solve CRA problems.

- volume 4, no. 2 (Spring 2012) 
In this experiment, participants' attentional focus was manipulated by engaging them in one of two tasks-a center-focused flanker task or a rapid object identification task-ostensibly demanding distinct types of visual attention (Skogsberg, 2008), and participants' verbal problem solving performance and the processing used to solve the verbal problems were measured both before and after the visual attentional manipulation. If engaging in a specific attention task influences the participants' subsequent problem solving efforts, then participants' performance on the CRA problems should also change. However, it is possible that simply engaging in any kind of intervening task induces a change in performance on CRA problems, not traceable to either attention task. If so, then performance on the CRA problems will change across both tasks but the participants should not alter in the proportion of problems that they solve with insight or analysis. In sum, the study was designed to examine changes in participants' CRA performance due to completing one of two different attention tasks, each demanding different attentional states.

Importantly, this experiment examines whether engaging in a purely visual task affects verbal problem solving performance. If so, it would demonstrate that a general attention component is shared across (or at least influences) both of these seemingly distinct behaviors. Previous research examining attentional priming effects on creative problem solving (Friedman et al., 2003) utilized overt instructions to attend or search within a given spatial extent. We did not explicitly instruct participants to attend widely or narrowly, but rather, gave them tasks in which they would perform better if they attend in a particular way, e.g. whether or not they focus their attention to filter out distracting input. Thus, one could not argue that any instructions to attend "broadly" or "narrowly" could somehow bias participants' solution ratings-for example, that being asked to attend "broadly" could lead participants report solving the problem with insight by "thinking broadly." Finally, we include a measure of baseline performance, to rule out pre-existing differences in participants' use of problem solving methods.

For the center-focused flanker task, participants responded to the center letter in a display while ignoring flanker letters (see Eriksen \& Eriksen, 1974; Rowe et al., 2007 for similar tasks). For the rapid object identification task, participants identified pictures of animals that were briefly presented and masked (for a similar task, see Bar \& Biederman, 1998). An additional experiment examined the effects of two other attention tasks, a multiple object tracking task and a global motion detection task, but yielded only weak effects, and will not be discussed further. For the multiple-object tracking task, participants attentively tracked four preselected moving objects in a field of 20 identical moving objects (for a similar task, see lordanescu, Grabowecky, \& Suzuki, 2009). Finally, for the global motion detection task, participants viewed a field of moving dots, a subset of which moved together in a coherent motion pattern whereas the remaining dots moved in random 
directions. The participants' task was to identify the type of coherent motion (radial vs. linear) in the field of moving dots (see Saenz, Buracas, \& Boynton, 2003 for a similar task).

Based upon the correlational studies of attentional styles and creativity, previous attentional priming findings (e.g. Friedman et al., 2003), and the literature about these tasks, we predicted that participants completing the center-focused flanker task would solve more problems with analytic methods. The flanker task requires participants to focus attention in a narrow spatial extent. People who tend to exhibit narrow and less-distractible attentional states tend to solve fewer problems with insight (Ansburg \& Hill, 2003). Furthermore, when participants are primed to focus their perceptual attention narrowly, they show a restricted scope of conceptual attention and diminished creativity (Friedman et al., 2003). Thus, we predict that the participants completing the center-focused task should solve more problems analytically in the second CRA set than in the first.

In contrast, we hypothesized that participants completing the rapid object identification task would solve more CRA problems with insight on the second set than on the first set. The rapid object identification task involves identifying visually degraded pictures (i.e. briefly flashed and masked), and a task involving visually degraded (spatially blurred) pictures has previously been correlated with good performance on problems that are most typically solved with insight (Schooler \& Melcher, 1995). There are at least two possible reasons for this relationship. Identifying briefly flashed stimuli could encourage broader spatial attention in order to detect distinctive features, which vary unpredictably in location across trials. Alternatively, identifying very rapidly presented objects could encourage participants to increase attention to internal associations weakly activated by the stimuli, over attention to the visual stimuli themselves (which vanish before becoming adequate to identify the object). Prior to problem presentation, $\mathrm{fMRI}$ signal in visual cortex correlates with subsequent analytic solving (Kounios et al., 2006), suggesting that external attention is more conducive to analytic than to insight solving. In contrast, when people are given biofeedback and asked to increase alpha power in the right posterior cortex (i.e. decrease attention to visual sensations), they show increased access to weak verbal associates (Haarmann, George, Smaliy, \& Dien, 2012, this issue).

\section{Methods}

\section{Participants}

Forty-one healthy, right-handed, native English-speaking undergraduates ( $53.6 \%$ female; $M_{\text {age }}=18.6 \pm 0.7$ years) were pseudo-randomly assigned to the two attention task conditions using their SAT Reading and Math scores to ensure that the groups did not differ on either SAT score. One participant in the rapid object identification condition was replaced because this participant solved very few problems $(<2.5 \mathrm{SD}$ from the mean performance

- volume 4, no. 2 (Spring 2012) 
for all participants in the experiment). All participants consented to participate in the study, which was approved by the Institutional Review Board at Northwestern University.

\section{Procedure}

Each participant completed one of two CRA problem sets (see below), followed by either the center-focused flanker or the rapid object identification task, followed by the other set of CRA problems. The attention tasks were each presented in four blocks, and participants received short breaks between blocks to reduce fatigue.

\section{CRA Problems}

Participants had a maximum of 15 seconds to solve each CRA problem, and indicated if they solved the problem by pressing a button and saying their answer aloud for the experimenter to score. If the participant could not solve the problem, the experiment would automatically proceed to the next problem without providing a solution. Immediately after participants provided a solution, they indicated, based on scripted instructions, whether they had solved the problem with "insight," with "analytic" processing, or with some "other" method. The "other" method was used primarily because participants sometimes indicate "insight" for solutions that they recognized rapidly, even though they did not experience impasse or restructuring. A protocol analysis of these "instantaneous recognition" solutions suggests that they are qualitatively different from insight solutions and should not be averaged in with them (Cranford \& Moss, 2012, this issue). The "other" category, which also included guesses, errors, or responses for which the participants could not decide, was rarely used, so for the most part these "other" solutions were removed from the analyses. The scripted instructions that described insight and analytic solutions were:

In this experiment having an insight means that although at first you did not know the solution, you suddenly get the correct answer without understanding exactly how you found it. You may have double-checked the solution just to confirm it, but the initial feeling was something like, "Oh! Of course that's the solution." Remember that insight solutions occur after a period of uncertainty, even if this uncertainty period is as brief as a second or two. So you might be unsure at first, but then the answer suddenly occurs to you.

Analytic solutions might involve strategies such as deliberately and consciously testing out different words until you find the one that is the solution. If asked, you would be able to report the steps or methods that you used to reach your solution or the fact that you used a strategy of some kind. So, in this way, these solutions are more incremental or methodical. 
After hearing these instructions, any questions that participants had about using the solution ratings were answered until the participant felt comfortable using the ratings. These descriptions of insight and analytic "noninsight" solutions are similar to those used in previous studies that found behavioral and neural differences between problems receiving different solution ratings (Bowden \& Jung-Beeman, 2003a; Jung-Beeman et al., 2004; Kounios et al., 2006, 2008; Subramaniam et al., 2009). Thus, any differences across solution types were expected to reflect underlying differences in information processing, rather than simply participants' preferences for each rating.

Participants received no feedback as to whether or not the answers they provided were correct. Only data from trials in which participants provided a correct response within the 15 second response window were analyzed because few incorrect responses were provided. The two sets of 40 CRA problems each were created using an items-analysis of solution data collected from a different sample of 80 undergraduate participants, so that the problems in each set were equivalent in difficulty and as equally likely to be solved with insight as possible.

On average, the problems in each set were solved equally often (Set $A=49.2 \%$ solution rate; Set $\mathrm{B}=49.2 \%$ solution rate), $\mathrm{t}(79)=.00, p=1.0, d=0.0,95 \% \mathrm{Cl}[49.1 \%, 49.4 \%]$, and the sets showed equal proportions of insight vs. "noninsight" ratings (Set $A=53.5 \%$ insight solutions; Set $\mathrm{B}=52.0 \%$, insight solutions), $\mathrm{t}(79)=.70, p=.49, d=0.16,95 \% \mathrm{Cl}$ $[53.4 \%, 53.7 \%]$. Set order was counter-balanced across participants, so that approximately half of the participants in each group saw Set A first and Set B second, and the other half saw the reverse. In the rapid object identification condition, ten participants received Set $A$ first and ten received Set B first. However, in the center-focused condition, eleven participants received Set $A$ first and nine received Set $B$ first. Nevertheless, there was no main effect of set, and the order of the sets did not interact reliably with any factor in our analyses, so the set order variable was dropped for all analyses. All statistical analyses were conducted in SPSS, and unless noted, all analyses utilize an alpha criterion of .05 for statistical significance.

\section{Attention Tasks}

Both attention tasks were programmed in Vision Shell Software (micro ML, Inc.) and were presented on a 17" CRT color monitor using a Macintosh (Power PC 8600/300). During each of the attention tasks, participants' head position was restrained with a chinrest positioned $65 \mathrm{~cm}$ from the display monitor.

In the center-focused flanker task, participants pressed a button to indicate the identity of a letter (either T or S) presented at fixation while ignoring two other flanking characters. The flankers were the opponent central item on interference trials (e.g. ST S), and were randomly generated numbers on neutral trials (e.g. $3 T 3$ or $4 S 4$ ). Half of the

- volume 4, no. 2 (Spring 2012) 
trials were interference trials and half were neutral trials. The central letter subtended $0.79^{\circ} \times 0.44^{\circ}$ visual angle from the center fixation point, and the flanker letters each subtended $1.41^{\circ}$ vertically $\times 0.97^{\circ}$ horizontally and were aligned horizontally with the center letter at $1.3 \mathrm{~cm}$ eccentricity (1.140 away from fixation). After a 1.5 second presentation of the central fixation point, the letters were presented for $214 \mathrm{~ms}$ in a black Helvetica font against an achromatic gray background. The trial terminated after the participant's button press, which initiated the next trial. After 10 practice trials to become used to the task, participants completed four blocks of 80 experimental trials each. For each participant, if any trial exceeded 3 standard deviations in reaction time (RT) from their within-task average, the trial (and excessive RT) was discarded.

In the rapid object identification task, participants verbally named a picture of an animal that was presented on the screen briefly $(40.26 \mathrm{~ms})$ and followed by a $215 \mathrm{~ms}$ random-dot mask. The stimuli were 28 gray-scale photographs of animals subtending $3.5^{\circ}$ $\times 3.5^{\circ}$ visual angle from center fixation presented against an achromatic gray background within a placeholder box. The masks were generated within the placeholder box using randomly assigned dot pixels ranging from black to white. Participants were informed that all of the experimental trials contained pictures of animals. Participants in the rapid object identification condition completed four blocks in which the 28 experimental trials were repeated. Before the first block of 28 experimental trials, participants received 10 practice trials containing nonliving artifacts.

Participants initiated each trial by pressing a button, with a delay of 134 ms between the button press and the onset of the picture. Response time was not limited; however, if participants hesitated for several seconds, the experimenter would encourage them to make their "best guess." After recording the participants' responses, the experimenter advanced to the next trial.

\section{Results}

\section{CRA Problem Performance}

The principal aim of our experiment was to examine whether participants changed the way they solved verbal problems after completing two different visual attention tasks. Indeed, participants' reports of their use of insight or analytic processing differed after each attention task, as revealed by a significant interaction in a $3 \times 2 \times 2$ ANOVA with Solution Type (insight, analysis, or other), Session (before vs. after), and Attention Task Group (center-focused flanker or rapid object identification) as factors, $F(2,76)=4.02, p=.022$, $\eta_{p}{ }^{2}=.096$ (see Table 1). There was a main effect of Session, $F(1,38)=15.85, p<.001, \eta_{p}{ }^{2}=$ .294, a main effect of Solution Type, $F(2,76)=28.86, p<.001, \eta_{p}^{2}=.432$, but no main effect of Attention Task Group by itself, $F(1,38)=0.003, p=.957, \eta_{p}^{2}<.001$. Planned contrasts 
revealed that participants solved more CRA problems with analytic processing after the center-focused flanker trials than they solved before them, $t(19)=2.81, p=.011, d=0.63$, $95 \% \mathrm{Cl}[0.75,5.15]$, whereas they did not change the number of problems solved with insight, $t(19)=-.98, \mathrm{p}=.341, d=.22,95 \% \mathrm{Cl}[-1.20,3.30]$. In contrast, participants solved more problems with insight after the rapid object identification trials than before them, $t(19)=2.12, p=.047, d=.47,95 \% \mathrm{Cl}[0.20,3.08]$, but did not change in the number of analytic solutions they produced, $t(19)=1.24, p=.23, \mathrm{~d}=.28,95 \% \mathrm{Cl}[-1.75,0.45]$. Participants in both groups did not change in the number of solutions labeled as "other", and most participants rarely used this category if they used it at all.

Table 1. Mean number (SEM) of CRA solutions by type before and after the attention tasks

\begin{tabular}{|c|c|c|c|c|c|c|c|c|}
\hline \multirow{2}{*}{$\begin{array}{l}\text { Attention } \\
\text { Task Group }\end{array}$} & \multicolumn{2}{|c|}{ Insight } & \multicolumn{2}{|c|}{ Analysis } & \multicolumn{2}{|c|}{ Other } & \multicolumn{2}{|c|}{ Total Solved } \\
\hline & Before & After & Before & After & Before & After & Before & After \\
\hline Center Focus & 6.9 & 7.9 & 7.3 & 10.2 & 1.4 & 1.5 & 15.5 & 19.6 \\
\hline Flanker $^{a}$ & (1.0) & $(1.3)$ & (0.9) & $(1.2)$ & $(0.4)$ & $(0.5)$ & $(0.9)$ & (1.1) \\
\hline Rapid Object & 10.1 & 11.7 & 5.3 & 4.7 & 1.4 & 1.8 & 16.8 & 18.1 \\
\hline Identification $^{\mathrm{a}}$ & (1.4) & $(1.5)$ & $(0.8)$ & $(0.9)$ & $(0.5)$ & $(0.7)$ & $(1.1)$ & (1.3) \\
\hline All & 8.5 & 9.8 & 6.3 & 7.4 & 1.4 & 1.6 & 16.1 & 18.8 \\
\hline Participants & (0.9) & $(1.0)$ & $(0.6)$ & $(0.8)$ & $(0.3)$ & $(0.4)$ & $(0.7)$ & $(0.8)$ \\
\hline
\end{tabular}

${ }^{\mathrm{a}} \mathrm{N}=20 ; \mathrm{CRA}=$ Compound Remote Associates

In addition, when removing the rarely-used "other" category from consideration and examining the percentage of insights produced, participants exhibited a reliable Attention Task Group by Session interaction, $F(1,38)=5.57, p=.024, \eta_{p}^{2}=.128$. There was a main effect of Attention Task Group, $F(1,38)=7.26, p=.010, \eta_{p}{ }^{2}=.160$, but no main effect of Session by itself, $F(1,38)=0.10, p=.76, \eta_{p}^{2}=.003$. Participants in the rapid object identification group showed a higher percentage of solutions via insight after $(M=69.4 \%, S E M=$ $5.3 \%)$ than before $(M=61.7 \%, S E M=5.5 \%)$ the task, $t(19)=2.28, p=.034, d=0.51,95 \% \mathrm{Cl}$ $[0.6 \%, 14.9 \%]$. However, the center-focused flanker group did not show a reliable change in the percentage of solutions produced by insight before $(M=47.4 \%, S E M=6.5 \%)$ vs. $\operatorname{after}(M=41.5 \%, S E M=6.2 \%)$ the task, $t(19)=1.26, p=.22, d=0.28,95 \% \mathrm{Cl}[-3.9 \%, 15.8 \%]$.

These changes in participants' reports of the type of processing after the attention tasks did not simply reflect changes in the way they labeled their solutions. A $2 \times 2$ Session by Attention Task Group ANOVA on the total number of correct responses revealed a trend toward an interaction with the interleaved attention task, $F(1,38)=4.03, p=.052$, $\eta_{p}{ }^{2}=.096$ (see Table 1). Participants' overall solving performance also improved from Set 
One to Set Two, as revealed by a reliable main effect of Session in this ANOVA, $F(1,38)=$ $15.85, p<.001, \eta_{p}^{2}=.294$. However, there was no reliable main effect of Attention Task Group by itself, $F(1,38)=0.003, p=.957, \eta_{p}^{2}<.001$. Planned contrasts revealed that the participants who completed the center-focused flanker task reliably improved their overall solving performance in the second session, $t(19)=3.73, p=.001, d=0.83,95 \% \mathrm{Cl}[1.80$, $6.40]$, but those who completed the rapid object identification task did not perform reliably better on Set Two, $t(19)=1.66, p=.11, d=0.37,95 \% \mathrm{Cl}[-0.36,3.06]$.

To rule out the possibility that baseline differences in solution processes were responsible for the differences observed after the attention tasks, post hoc Tukey's HSD tests were conducted in SPSS on participants' data from Set One to assess whether the two groups showed different solution rates or differed in the number of solutions labeled as insight, analysis, or other at baseline. These tests revealed no statistically reliable differences between the groups on Set One in overall solving rates, $t(38)=0.93, p=.49, d$ $=0.29,95 \% \mathrm{Cl}[-1.44,4.04]$, the number of solutions via analysis, $t(38)=1.65, p=.12, d=$ $0.52,95 \% \mathrm{Cl}[-0.37,4.27]$, and the number of solutions labeled as "other", $t(38)=0.0, p=$ $1.0, d=0.0,95 \% \mathrm{Cl}[-1.23,1.23]$. However, the Tukey's HSD tests did reveal a trend toward a greater number of insight solutions in the rapid object identification group than the center-focused flanker group during Set One, $t(38)=1.84, p=.071, d=0.58,95 \% C l[-0.22$, 6.72]. Nevertheless, when the number of insight solutions was expressed as a percentage of the total solutions (excluding solutions labeled as "other"), HSD tests revealed that the two groups did not reliably differ at baseline, $t(38)=1.68, p=.11, d=0.55,95 \% \mathrm{Cl}[-2.4 \%$, $31.0 \%]$. Thus, the changes in solution ratings were unlikely to be the result of general, pre-existing differences between the groups in the processing they used to solve the CRA problems.

Table 2. Mean (SEM) reaction time (in sec) of solutions by type before and after attention tasks

\begin{tabular}{|c|c|c|c|c|c|c|c|c|}
\hline \multirow{2}{*}{$\begin{array}{c}\text { Attention } \\
\text { Task Group }\end{array}$} & \multicolumn{2}{|c|}{ Insight RT (s) } & \multicolumn{2}{|c|}{$\begin{array}{c}\text { Analysis RT } \\
\text { (s) }\end{array}$} & \multicolumn{2}{|c|}{ "Other" RT (s) } & \multicolumn{2}{|c|}{$\begin{array}{c}\text { All Correct RT } \\
\text { (s) }\end{array}$} \\
\hline & Before & After & Before & After & Before & After & Before & After \\
\hline \multirow{2}{*}{$\begin{array}{l}\text { Center Focus } \\
\text { Flanker }^{\mathrm{a}}\end{array}$} & 6.66 & 6.58 & 7.64 & 7.77 & 6.78 & 4.54 & 6.86 & 6.79 \\
\hline & $(0.5)$ & $(0.6)$ & $(0.4)$ & $(0.3)$ & (1.3) & $(0.8)$ & $(0.3)$ & $(0.2)$ \\
\hline \multirow{2}{*}{$\begin{array}{l}\text { Rapid Object } \\
\text { Identification }^{\mathrm{a}}\end{array}$} & 7.02 & 6.47 & 8.12 & 8.16 & 6.28 & 8.33 & 7.05 & 6.54 \\
\hline & (0.4) & $(0.4)$ & $(0.5)$ & $(0.4)$ & (1.3) & $(2.3)$ & $(0.3)$ & $(0.3)$ \\
\hline \multirow{2}{*}{ All Participants } & 6.84 & 6.52 & 7.86 & 7.95 & 6.54 & 6.34 & 6.96 & 6.67 \\
\hline & $(0.3)$ & (0.3) & $(0.3)$ & $(0.2)$ & (0.9) & (1.2) & $(0.2)$ & $(0.2)$ \\
\hline
\end{tabular}

${ }^{\mathrm{a}} \mathrm{N}=20 ; \mathrm{CRA}=$ Compound Remote Associates; $\mathrm{RT}$ = reaction time

All reaction times (RTs) for correctly solved problems (in seconds) can be found in Table 2 . A $3 \times 2 \times 2$ ANOVA revealed no reliable three way interaction between Solution 
Type, Session, and Attention Task Group for the RT data, $F(2,24)=0.10, p=.91, \eta_{p}{ }^{2}=.008$. On average, both groups solved the CRA problems in the same amount of time, $F(1,12)=$ $0.87, p=.37, \eta_{p}{ }^{2}=.008$, which did not reliably differ before and after the attention tasks, $F(1,12)=1.44, p=.25, \eta_{p}^{2}=.107$. However, problems that were rated differently (i.e. insight, analysis, other) showed different RTs when averaged across both sessions, [mean insight $\mathrm{RT}=6.82 \mathrm{sec}$, $($ SEM $=.46 \mathrm{sec})$; mean analysis $\mathrm{RT}=8.08 \mathrm{sec}($ SEM $=.39 \mathrm{sec})$; mean "other" $\mathrm{RT}=5.49 \mathrm{sec},(S E M=.67 \mathrm{sec})] ; F(2,24)=8.30, p=.002, \eta_{p}{ }^{2}=.409$. When comparing RTs averaged across sessions for each solution type, solutions by analysis were slower overall than solutions by insight; weighted RT insight $=6.45 \mathrm{sec}$, (SEM $=0.28 \mathrm{sec})$, weighted RT analysis $=7.89$, SEM $=0.24 \mathrm{sec}, t(34)=4.92, p<.001, d=0.68,95 \% \mathrm{Cl}[0.71,2.16]$. (Some participants produced no insight or analysis solutions so $d f<39$; no other differences were found). In addition, there were no main effects or interactions when RTs for insight and analysis solutions were considered without including the solutions labeled as other.

Table 3. Mean (SEM) reaction time or accuracy in each attention task condition across blocks

\begin{tabular}{ccccc} 
& \multicolumn{2}{c}{ Center-Focused Flanker $^{\mathrm{a}}$} & & Rapid Object Identification $^{\mathrm{a}}$ \\
\cline { 2 - 3 } Block & Overall RT & \% Correct & & \% Correct \\
\hline \multirow{2}{*}{1} & $512 \mathrm{~ms}$ & $95 \%$ & $20 \%$ \\
& $(17)$ & $(1 \%)$ & $(3 \%)$ \\
2 & $508 \mathrm{~ms}$ & $97 \%$ & $24 \%$ \\
& $(17)$ & $(1 \%)$ & $(4 \%)$ \\
3 & $497 \mathrm{~ms}$ & $97 \%$ & $26 \%$ \\
& $(15)$ & $(1 \%)$ & $(4 \%)$ \\
\multirow{2}{*}{4} & $493 \mathrm{~ms}$ & $94 \%$ & $28 \%$ \\
& $(16)$ & $(3 \%)$ & $(4 \%)$ \\
\hline
\end{tabular}

${ }^{\mathrm{a}} \mathrm{N}=20 ; \mathrm{RT}=$ reaction time

\section{Attention Task Performance}

In addition to evidence that the attention tasks altered the types of processes each group used to solve the CRA problems, there is also evidence that the attention tasks each altered the participants' attentional states, such that they grew increasingly adept at the type of attention necessary for the task (see Table 3). Participants in the rapid object identification group identified, on average, more pictures in the fourth task block $(27.5 \%$, SEM $=3.9 \%)$ than in the first $(19.8 \%, \operatorname{SEM}=3.3 \%), t(19)=4.56, p<.001, d=1.02,95 \% \mathrm{Cl}$ $[4.2 \%, 11.2 \%]$. What makes this improvement remarkable is the difficulty of the task due to the extremely short $(40 \mathrm{~ms}$ ) exposure time and the backward masking, as well as most participants' insistence that they could not identify anything correctly. In a similar vein, participants completing the center-focused flanker task reliably responded more quickly 
in the fourth block (mean RT $=493 \mathrm{~ms}, \mathrm{SEM}=16 \mathrm{~ms}$ ) than in the first block (mean RT= $511 \mathrm{~ms}, \mathrm{SEM}=17 \mathrm{~ms}), t(19)=2.13, p=.046, d=0.48,95 \% \mathrm{Cl}[0.3,36.9]$. This decrease was reliable for interference trials, $t(19)=2.24, p=.037, d=0.50,95 \% \mathrm{Cl}[1.3,38.3]$ and a trend level for neutral trials, $t(19)=1.85, p=.080, d=0.41,95 \% \mathrm{Cl}[-2.3,37.1]$, reflecting the fact that as participants improved on the task, most of the improvement came from faster responses to interference trials. Despite these faster response times, participants did not show reliable decreases in accuracy between the first block (95.4\%, SEM $=1.1 \%)$ and the fourth block $(93.5 \%, S E M=3.0), t(19)=0.59, p=.560, d=0.13,95 \% \mathrm{Cl}[-4.7 \%$, 8.4\%]. (They also showed no reliable decreases in accuracy on interference or neutral trials considered separately.) Thus, both groups were improving in performance on their attention task prior to beginning the second set of CRA problems, further supporting the notion that the task they completed influenced their performance.

\section{Discussion}

Participants' ability to solve CRA problems and their perception of the manner in which they solved them both changed after performing the different attention tasks interleaved between the two CRA problem sets. Completing the rapid object identification task and the center-focused flanker task caused participants to improve on the second set of CRA problems, but in different ways. Compared to their baseline performance, participants solved more problems via insight after the rapid object identification task, and more problems via analysis following the center-focused flanker task. It is particularly striking that performing these visual tasks affected participants' subsequent verbal problem solving, suggesting that the attention processes involved are quite general.

These results suggest that two different visual attention tasks successfully altered participants' attentional state, facilitating verbal problem solving in two distinct ways. As predicted, the center-focused flanker task narrowed participants' attentional focus because they needed to filter out the distracters, which led to an increase in the number of analytic solutions that they produced. In previous research, participants who were better at screening out distractors, when instructed to do so, solved more focal anagrams and remembered fewer distracting anagram solutions, compared to participants who attended to stimuli they were instructed to ignore (Ansburg \& Hill, 2003). The results of the current study show that simply completing a perceptual task that requires one to focus can yield more incremental, analytic solutions to verbal problems.

In contrast, and also as predicted, participants solved more problems with insight following the rapid object identification task, echoing previously described correlations, wherein people who perform well at identifying visually degraded pictures also perform well on verbal problems typically solved with insight (Schooler \& Melcher, 1995). One possibility is that the rapid object identification task broadened participants' attentional 
focus because they needed to attend to all parts of the briefly presented stimulus in order to gain an impression of its identity, and they could not know beforehand which part of the image would contain the most distinctive information. Broadening spatial attention could have led to broadened conceptual attention, which in turn helped participants attend to non-prepotent CRA solution candidates (Rowe et al., 2007), leading them to solve more problems with insight.

Alternatively, the rapid object identification task could facilitate insight by encouraging people to detect and subsequently attend to weakly activated internal representations, rather than only attending to external stimuli or strong internal representations. That is, in the absence of strong external stimuli during the rapid object identification task, the participants may have to attend (less selectively) to weak sub-threshold internal processing in order to correctly identify the stimulus. Subsequently, while solving CRA problems, attending to sub-threshold activation of distant associations to the problem words may be conducive to insight solutions. This interpretation is consistent with prior research in which, just before participants solved CRAs with insight, they appeared to engage in gating or attenuation of visual input, as marked by alpha band activity over right occipital parietal lobe, immediately preceded insight but not analytic solutions (Jung-Beeman et al., 2004). Moreover, before even reading a new problem, activity in visual cortex was stronger prior to problems subsequently solved analytically than prior to problems subsequently solved with insight. It is also possible that both attentional broadening and attention to weak internal associations played a role in increasing insight solutions.

The rapid object identification task is also different from many typical attention tasks, such the center-focused flanker, in that it is open-ended, (i.e. the choice involved in the task was not a binary one, as any animal could be a potential solution). Because the external perceptual evidence in this task is highly degraded, participants adopting a broadened mindset would be more likely to consider answers that are consistent with the weak perceptual evidence. This broadened mindset could have subsequently facilitated solving problems in the CRA problem set with insight. It is also possible that a combination of spatially broadened attentional states, attention to weak associations, and the openendedness of the task led to the increase in insight solutions, and follow up studies could investigate the role of each these factors systematically.

For several reasons, it is unlikely that participants simply improved at solving the CRA problems over time, or would have following any intervening task. First, the two tasks caused distinct changes in the manner in which the extra problems were solved, with the center-focused task leading to increased analytic solutions, and the rapid object identification task leading to increased insight solutions. In addition, in prior studies participants showed no increase in solution or insight judgments across many sets of CRA problems (Bowden \& Beeman, 1998; Bowden \& Jung-Beeman, 2003a; Jung-Beeman et al., 2004;

- volume 4, no. 2 (Spring 2012) 
Kounios et al., 2006; Subramaniam et al., 2009). Finally, the attention tasks themselves were fairly demanding and lasted approximately 20-30 minutes depending on the participant. Thus, potential practice effects might be mitigated by participants' fatigue from completing four blocks of demanding attention tasks. Thus, these results are consistent with the notion that the two attentional tasks modified participants' attentional state and improved verbal problem-solving performance via a particular type of processing.

Although the possibility cannot be entirely eliminated, it is unlikely that the changes in reported manner of solving reflect only a shift in criteria for reporting insight versus analysis. As noted in the introduction, a variety of behavioral (Bowden \& Jung-Beeman, 2003a) and neural correlates (Aziz-Zadeh et al., 2009; Jung-Beeman et al., 2004; Kounios et al., 2006; 2008; Subramaniam et al., 2009) all demonstrate that different solving processes are engaged prior to people's self-report of solving by insight or by analysis. In the current experiment, both groups, before and after the attention task, showed the same difference in solving time, solving problems slightly faster when reporting insight than when reporting analysis. Nevertheless, the finding that insight solutions were faster than analysis solutions, with no differences across groups or time, does not mean that insight solutions merely represent fast solutions, as there is considerable overlap between the distributions of the RTs for insight and analysis solutions. In addition, this difference in RT has been found even when objective neural differences are found between insight and analysis solutions (e.g. Kounios et al., 2006; Subramaniam et al., 2009). Finally, the difference between RTs for insight, analysis, and other solutions did not vary by group, ruling out the possibility that the divergent changes in solution types across sessions between the two groups was caused by a difference in the distribution of RTs.

In sum, we have found that performing different visual attention tasks alters people's attentional state, affecting subsequent verbal problem processing and solving performance. There may be several ways in which insight and analysis solutions differ, and a full review is beyond the scope of this paper. However, the current results suggest that people's general attentional state before attempting to solve a verbal problem influences the processes engaged during the solving effort, and thus shifts the likelihood that the solution is attained either through insight or through analysis. The most plausible mechanism to explain the current results is, simply put, that engaging in different visual tasks alters the degree to which people focus their attention on the strongest inputs and associations (while likely inhibiting weaker ones). Engaging in a task that requires attending to weakly active perceptual associations (in the rapid identification task) subsequently improves people's ability to detect weakly active semantic associations. Solving with insight requires restructuring or a shift in interpretation, and improved ability to detect weakly activated associations improves the ability to shift interpretations, and thus facilitates solving by insight. 
This research extends previous correlational work that examined individual differences in attention and creative insight. These studies suggest that creative individuals often exhibit states of broadened attention and that a broadened state of attention is sometimes useful in order to complete divergent thinking tasks and to solve problems with insight (Ansburg \& Hill, 2003; Carson, Peterson, \& Higgins, 2003; Dykes \& McGhie, 1976; Kasof, 1997; Mendelsohn \& Griswold, 1966; Mendelsohn \& Lindholm, 1972; Necka, 1999; Rawlings, 1985; Rowe et al., 2007; Schooler \& Melcher, 1995). However, it was unclear in previous studies whether these broadened attention states were diffuse sensory states (e.g. visuospatial, auditory) or if they were diffuse conceptual states, or both. Our research suggests that a general attention state (influencing both conceptual and visuospatial attention) affects the manner in which people solve problems. Focused attention states facilitate the use of analysis, whereas diffuse attention states facilitate the use of insight.

Although general problem solving skills support both creative and analytic thought (Ansburg, 2000), creative thought may rely more on diffuse states of attention than on focused states of attention because incidental cues provide more benefit to creative problem solving efforts, whereas these incidental cues simply distract from analytic problem solving efforts (Seifert et al., 1995). However, it is also important to note that broadened or defocused attention by itself does not automatically lead to creative thought. Even though creative solutions to problems may require people to think broadly, successful solvers must eventually choose the most appropriate solution to the problem. Extremely defocused attention without some degree of focusing could lead to novel but nonfunctional ideas such as those produced by schizophrenic patients (Dykes \& McGhie, 1976), so effective creative thought requires some divergent thinking and some convergent thinking (Cropley, 2006). Therefore, habitually defocused attention must be coupled with the ability to control one's focus of attention on practical ideas to arrive at a novel, but also appropriate or workable solution.

Our study demonstrates that, within individuals, the state of attention can vary, and be manipulated, to favor one form of solving over another. In addition to biofeedback, which has been shown to help people increase access to remote verbal associates (Haarmann et al., 2012, this issue), there may be other ways to manipulate attention states, or to give people more control over their own attention states. For instance, a single session of hypnosis can improve highly-hypnotizable participants' executive performance, specifically by eliminating interference on the color-word version of the Stroop task (Raz, Fan, \& Posner, 2005). In addition, participants who received sessions of integrated mindbody training over a period of five days showed marked improvements on executive functioning tasks when compared to a group of participants who received relaxation training (Tang et al., 2007). It remains to be seen whether such training would improve individuals' performance on insight or analytic problems, or both.

- volume 4, no. 2 (Spring 2012) 
Focusing on the role that different attentional states play in creative problem solving will lead to a deeper understanding of the cognitive and neural bases of creativity and insight. Understanding how attention and task demands modulate these processes could explain why some physical and social environments are more conducive to creative insights while others are less so. Research based on careful attentional manipulations could lead to better design of research labs, workplaces, and classrooms to better promote creativity and innovative thinking.

\section{References}

Ansburg, P. I. (2000). Individual differences in problem solving via insight. Current Psychology, 19(2), 143-146.

Ansburg, P. I., \& Hill, K. (2003). Creative and analytic thinkers differ in their use of attentional resources. Personality and Individual Differences, 34(7), 1141-1152.

Aziz-Zadeh, L., Kaplan, J. T., \& lacoboni, M. (2009). "Aha!": The neural correlates of verbal insight solutions. Human Brain Mapping, 30, 908-916.

Bar, M., \& Biederman, I. (1998). Subliminal visual priming. Psychological Science, 9(6), 464469.

Beeman, M. J., \& Bowden, E. M. (2000). The right hemisphere maintains solution-related activation for yet-to-be-solved problems. Memory \& Cognition, 28(7), 1231-1241.

Bowden, E. M., \& Beeman, M. J. (1998). Getting the right idea: Semantic activation in the right hemisphere may help solve insight problems. Psychological Science, 9(6), 435440.

Bowden, E. M., \& Jung-Beeman, M. (2003a). Aha! - Insight experience correlates with solution activation in the right hemisphere. Psychonomic Bulletin \& Review, 10(3), 730-737.

Bowden, E. M., \& Jung-Beeman, M. (2003b). Normative data for 144 compound remote associate problems. Behavior Research Methods Instruments \& Computers, 35(4), 634-639.

Bowden, E. M., Jung-Beeman, M., Fleck, J., \& Kounios, J. (2005). New approaches to demystifying insight. Trends in Cognitive Sciences, 9(7), 322-328.

Carson, S. H., Peterson, J. B., \& Higgins, D. M. (2003). Decreased latent inhibition is associated with increased creative achievement in high-functioning individuals. Journal of Personality and Social Psychology, 85(3), 499-506.

Cranford, E. A. \& Moss, J. (2012). Is insight always the same? A protocol analysis of insight in compound remote associate problems. The Journal of Problem Solving, 4(2), Article 7.

Cropley, A. (2006). In praise of convergent thinking. Creativity Research Journal, 18(3), 391-404.

Duncker, K. (1945). On problem solving. Psychological Monographs, 58(5, Whole No. 270). 
Dykes, M., \& McGhie, A. (1976). A comparative study of attentional strategies of schizophrenic and highly creative normal subjects. British Journal of Psychiatry, 128, 50-56.

Eriksen, B. A., \& Eriksen, C. W. (1974). Effects of noise letters upon the identification of a target letter in a nonsearch task. Perception and Psychophysics, 16(1), 143-149.

Friedman, R. S., Fishbach, A., Förster, J., \& Werth, L. (2003). Attentional priming effects on creativity. Creativity Research Journal, 15(2-3), 277-286.

Gasper, K. (2003). When necessity is the mother of invention: Mood and problem solving. Journal of Experimental Social Psychology, 39(3), 248-262.

Gasper, K. (2004a). Do you see what I see? Affect and visual information processing. Cognition \& Emotion, 18(3), 405-421.

Gasper, K. (2004b). Permission to seek freely? The effect of happy and sad moods on generating old and new ideas. Creativity Research Journal, 16(2-3), 215-229.

Gasper, K., \& Clore, G. L. (2002). Attending to the big picture: Mood and global versus local processing of visual information. Psychological Science, 13(1), 34-40.

Haarmann, H. J., George, T., Smaliy, A. \& Dien, J. (2012). Remote associates test and alpha brain waves. The Journal of Problem Solving, 4(2), Article 4.

Iordanescu, L., Grabowecky, M., \& Suzuki, S. (2009). Demand-based dynamic distribution of attention and monitoring of velocities during multiple-object tracking. Journal of Vision, 9(4):1-12, http://journalofvision.org/9/4/1/, doi:10.1167/9.4.1.

Isen, A. M. (1999). On the relationship between affect and creative problem solving. In S. W. Russ (Ed.), Affect, creative experience, and psychological adjustment (pp. 3-17). Philadelphia, PA: Brunner/Mazel.

Isen, A. M., Daubman, K. A., \& Nowicki, G. P. (1987). Positive affect facilitates creative problem-solving. Journal of Personality and Social Psychology, 52(6), 1122-1131.

Jung-Beeman, M., Bowden, E. M., Haberman, J., Frymiare, J. L., Arambel-Liu, S., Greenblatt, R., et al. (2004). Neural activity when people solve verbal problems with insight. PLoS Biology, 2(4), 500-510.

Kasof, J. (1997). Creativity and breadth of attention. Creativity Research Journal, 10(4), 303-315.

Kaufmann, G. (2003). Expanding the mood - creativity equation. Creativity Research Journal, 15(2-3), 131-135.

Kaufmann, G., \& Vosburg, S. K. (1997). "Paradoxical" mood effects on creative problemsolving. Cognition \& Emotion, 11(2), 151-170.

Kerns, J. G., Cohen, J. D., MacDonald, A. W., Cho, R. Y., Stenger, V. A., \& Carter, C. S. (2004). Anterior cingulate conflict monitoring and adjustments in control. Science, 303(5660), 1023-1026.

Kounios, J., Frymiare, J. L., Bowden, E. M., Fleck, J. I., Subramaniam, K., Parrish, T. B., et al. (2006). The prepared mind - Neural activity prior to problem presentation predicts subsequent solution by sudden insight. Psychological Science, 17(10), 882-890.

- volume 4, no. 2 (Spring 2012) 
Kounios, J., Fleck, J. I., Green, D. L., Payne, L., Stevenson, J. L., Bowden, E. M., et al. (2008). The origins of insight in resting-state brain activity. Neuropsychologia, 46(1), 281-291.

Maier, N. R. F. (1931). Reasoning in humans: II. The solution of a problem and its appearance in consciousness. Journal of Comparative Psychology, 12 181-194.

Mednick, S. A., \& Mednick, M.T. (1967). Examiners'Manual Remote Associations Test. Boston, MA: Houghton Mifflin.

Mendelsohn, G. A., \& Griswold, B. B. (1966). Assessed creative potential, vocabulary level, and sex as predictors of use of incidental cues in verbal problem solving. Journal of Personality and Social Psychology, 4(4), 423-431.

Mendelsohn, G. A., \& Lindholm, E. P. (1972). Individual differences and role of attention in use of cues in verbal problem-solving. Journal of Personality, 40(2), 226-241.

Metcalfe, J., \& Wiebe, D. (1987). Intuition in Insight and Noninsight Problem-Solving. Memory \& Cognition, 15(3), 238-246.

Necka, E. (1999). Creativity and attention. Polish Psychological Bulletin, 30(3), 85-97.

Perkins, D. (2001). The Eureka Effect: The Art and Logic of Breakthrough Thinking. New York, New York: W. W. Norton \& Company.

Rawlings, D. (1985). Psychoticism, creativity and dichotic shadowing. Personality and Individual Differences, 6(6), 737-742.

Raz, A., Fan, J., \& Posner, M. I. (2005). Hypnotic suggestion reduces conflict in the human brain. Proceedings of the National Academy of Sciences of the United States of America, 102(28), 9978-9983.

Rowe, G., Hirsh, J. B., \& Anderson, A. K. (2007). Positive affect increases the breadth of attentional selection. Proceedings of the National Academy of Sciences of the United States of America, 104(1), 383-388.

Saenz, M., Buracas, G. T., \& Boynton, G. M. (2003). Global feature-based attention for motion and color. Vision Research, 43(6), 629-637.

Schooler, J. W., \& Melcher, J. (1995). The ineffability of insight. In S. M. Smith, T. B. Ward \& R. A. Finke (Eds.), The Creative Cognition Approach (pp. 97-133). Boston, MA:The MIT Press.

Schooler, J. W., Ohlsson, S., \& Brooks, K. (1993). Thoughts beyond words: When language overshadows insight. Journal of Experimental Psychology-General, 122(2), 166-183.

Seifert, C. M., Meyer, D. E., Davidson, N., Patalano, A. L., \& Yaniv, I. (1995). Demystification of cognitive insight: Opportunistic assimilation and the prepared-mind perspective. In R. J. Sternberg \& J. E. Davidson (Eds.), The Nature of Insight (pp. 65-124). Boston, MA: MIT Press.

Skogsberg, K. (2008). Multiple modes of voluntary visual attention: Analysis of within test reliability, between group differences, and the interrelationships among tests of voluntary visual attention. (Doctoral dissertation). Available from ProQuest Dissertations and Theses database. (AAT 3331156). 
Sternberg, R. J., \& Davidson, J. E. (1995). The Nature of Insight. Boston, MA: MIT Press.

Subramaniam, K., Kounios, J., Parrish, T. B., \& Jung-Beeman, M. (2009). A brain mechanism for facilitation of insight by positive affect. Journal of Cognitive Neuroscience, 21(3), 415-432.

Tang, Y. Y., Ma, Y. H., Wang, J., Fan, Y. X., Feng, S. G., Lu, Q. L., et al. (2007). Short-term meditation training improves attention and self-regulation. Proceedings of the National Academy of Sciences of the United States of America, 104, 17152-17156.

Weisberg, R. W. (1986). Creativity, genius, and other myths. New York:W. H. Freeman.

Weissman, D.H., Gopalakrishnan, A., Hazlett, C.J., \& Woldorff, M.G. (2005). Dorsal anterior cingulate cortex resolves conflict from distracting stimuli by boosting attention toward relevant events. Cerebral Cortex, 15, 229-237.

\section{Acknowledgments}

MB was supported by grant 24467 from the John Templeton Foundation during the preparation of this manuscript. We would like to thank Michael Rock, Michael Claffey, and Hilary Johnson for help in running participants, and Lucica lordanescu and KatieAnn Skogsberg for help with setting up the attention tasks. 